\title{
Nivel de Exposición Sonora de Disparos en Campo Libre: Cálculo y Comprobación Experimental
}

\author{
Sound Exposure Level in Outdoors Gunshots: Calculation and Experimental Validation \\ Leandro S. Rodiño \\ I+A Ingeniería Acústica
}

Av. San Juan 3625, Oficina 37, Ciudad Autónoma de Buenos Aires, Argentina

ingenieria@ingenieriacustica.com

Recibido: 13/11/18; Aceptado: 07/02/19

\begin{abstract}
The acoustic analysis of gunshots provides valuable information in the forensic field. Through the measurement and calculation of the physical properties produced by the deflagration of the gases at the moment of shooting, it is possible to estimate the released acoustic energy. First, the calculation of the angular source energy proposed in the ISO 17201 standard is applied. Subsequently, the estimation of the acoustic propagation in free field is carried out to obtain the sound exposure level. Finally, a field test is performed in order to validate the simulated values. The implementation of the method is compiled in a practical software to automate a quick data loading and obtaining results.
\end{abstract}

Keywords: gunshots; acoustic; forensic science.

\begin{abstract}
Resumen - El análisis acústico de disparos realizados por armas de fuego permite obtener valiosa información en el ámbito forense. A través de la medición y el cálculo de diversas propiedades físicas producto de la deflagración de los gases ocurrida al momento de realizar un disparo es posible estimar la energía acústica liberada. En primera instancia se aplica el cálculo de la energía angular de la fuente propuesto en la norma ISO 17201. Posteriormente se realiza la estimación de la propagación acústica en campo libre para obtener el nivel de exposición sonora a distancias determinadas alrededor de la fuente. Por último, se realiza una prueba de campo con la finalidad de validar los valores simulados. La implementación del método se compila en un software práctico para automatizar una rápida carga de datos y obtención de resultados.
\end{abstract}

Palabras clave: disparos; acústica; ciencia forense.

\section{INTRODUCCIÓN}

La importancia del impacto que produce el sonido de disparos de armas de fuego, sobre una comunidad que se encuentre en zonas aledañas a áreas de tiro, cobra una vital importancia en sociedades que prevalecen la salud y el confort de sus habitantes [1]. Las áreas de tiro pueden tener fines recreativos como también así fines de entrenamiento militar del personal de las Fuerzas de Seguridad o Defensa. Para ambos casos existen actualmente manuales $y$ protocolos para preservar la calidad ambiental (acústica incluída) de dichas áreas [2].

Asimismo, en los últimos años, con el advenimiento y popularidad de los dispositivos digitales portátiles, ha incrementado considerablemente la recolectación de evidencia forense de sonidos de disparos por parte de testigos ocasionales [3]. Mediante la grabación de audio y video (mediante cámaras de vigilancia, celulares, etc) se ha generado un potencial material de análisis que en muchos casos puede aportar información valiosa a una causa a raíz del registro y posterior procesamiento de la señal acústica de un disparo, como por ejemplo en [4].

Dependiendo de la normativa utilizada, se puede cuantificar y caracterizar la energía acústica de un disparo mediante distintos parámetros. Dada la probada correlación entre el nivel de exposición sonora y la molestia percibida en las personas producto del sonido de disparos [5], el presente trabajo toma como referencia la ISO 17201-2 [6].

El objetivo de este trabajo es implementar un modelo teórico que permita estimar niveles de presión sonora producidos por disparos de armas de fuego en campo libre utilizando únicamente sus propiedades físicas (como masa de la pólvora y velocidad del proyectil). Mediante la posterior medición en campo de distintas situaciones simuladas se pretenden validar los resultados obtenidos.

\section{MARCO TEÓRICO}

En esta sección se describirán los principios del modelo teórico utilizado para la implementación del código computacional.

La deflagración de los gases producida al momento de realizar un disparo libera una cierta cantidad de energía química. Para la situación en que la medición empírica de dicha liberación energética no pueda realizarse, es posible estimarla utilizando las leyes de conservación de la energía en función de: la masa del propelente detonado y un valor de energía química específica, o de la velocidad de salida del proyectil en la boca del cañón, su masa y un porcentaje de conversión de energía cinética. Una parte de la energía química total, ya sea medida o calculada, se convierte luego en energía gaseosa (alrededor del $45 \%$ ) que posteriormente se transforma en energía acústica (4\% como valor de referencia) [7].

Conocida la energía acústica total, es posible aplicar una corrección direccional sobre el plano de distribución energético horizontal dependiendo del tipo de arma utilizada (revolver, rifle o pistola) y su espectro por bandas de frecuencia. La distribución espectral de la energía responde 
a la fórmula de densidad de Weber [8] que se muestra a continuación:

$$
E_{d}=\frac{P_{w}^{2}}{\rho c} \frac{1}{\pi} \int_{\omega_{1}}^{\omega_{2}}\left[\omega^{2}+9 \frac{c^{2}}{R^{2}}\left(\frac{c^{2}}{R^{2} \omega^{2}}+1\right)\right]^{-1} d \omega
$$

donde $E_{d}$ es la densidad de la energía acústica irradiada a través de una unidad de área desde la fuente hacia una dirección considerada entre las bandas $\omega_{1}$ y $\omega_{2}, P_{w}$ es una constante de presión (presión de Weber), $\rho$ es la densidad del aire, $c$ es la velocidad del sonido en el aire, $\omega$ es la frecuencia angular y $R$ es el radio de Weber (calculado a partir de la transformación energética del disparo). La integración por banda de frecuencias se debe realizar para cada ángulo de interés y así obtener finalmente la energía angular de la fuente. Definiendo los límites de integración se pueden establecer las bandas de análisis, ya sea el espectro completo o por bandas de diversas fracciones de octava.

La energía angular de la fuente es la magnitud necesaria para utilizar en el modelo de propagación acústica en campo libre y obtener el nivel de exposición sonora en un punto dado en relación a la ubicación y orientación conocidas del disparador. El modelo de cálculo de propagación (por atenuación sonora) se encuentra detallado en la ISO 172013 [9] y tiene lineamientos similares a los utilizados en la ISO 9613-2 [10], con las correcciones correspondientes según el caso. A continuación se muestra la fórmula general de cálculo:

$$
\begin{aligned}
& L_{E}(f)=L_{q}(\alpha, f)-A_{d i v}(r)+11 d B- \\
& A_{a t m}(r, f)-A_{b a r}(r, f)-A_{g r}(r, f)- \\
& A_{z}(r, f)-A_{\text {misc }}(r, f)
\end{aligned}
$$

donde $L_{E}$ es el nivel de exposición sonora en el punto de interés, $L_{q}$ es la energía angular de la fuente, $A_{d i v}$ es la atenuación por divergencia geométrica, $A_{a t m}$ es la atenuación por absorción del aire, $A_{b a r}$ es la atenuación debido al apantallamiento producido por un objeto, $A_{g r}$ es la atenuación producida por el suelo, $A_{z}$ es la atenuación en condiciones metereológicas no estándares y $A_{\text {misc }}$ es la atenuación debido a otros efectos misceláneos, todo expresado en decibelios, en función de una distancia $r$, una frecuencia $f$ y un ángulo de incidencia $\alpha$, según corresponda.

Es natural pensar una analogía entre la energía angular y la potencia acústica de una fuente, y entre el nivel de presión sonora y el nivel de exposición sonora. En ambos casos, la energía angular y la potencia acústica, son magnitudes inherentes a la fuente y pueden ser estimadas teóricamente utilizando propiedades físicas de la misma o inferidas indirectamente mediante la medición de otro parámetro y un posterior cálculo. En el caso de la potencia acústica, es usual medir el nivel de presión sonora de una fuente en un campo libre $o$ en condiciones acústicas conocidas y controladas para poder calcularla. Del mismo modo, para obtener la energía angular de un disparo se puede realizar la anteriormente mencionada estimación teórica o se puede medir el nivel de exposición sonora en un punto conocido y obtener de manera inversa el valor de la energía angular de la fuente utilizando la ecuación (2).
El método teórico descrito en esta sección es válido para estimar el nivel de energía angular de armas de fuego con calibres de hasta $20 \mathrm{~mm}$ y no más de $50 \mathrm{~g}$ de pólvora [6].

Se puede notar que la utilización del nivel de exposición sonora como parámetro de caracterización de un evento sonoro impulsivo de gran energía resulta especialmente adecuado debido a que los valores obtenidos no se ven modificados sustancialmente respecto de los distintos tiempos de integración que se pudieran llegar a utilizar.

$$
S E L=10 \log \left[\int_{0}^{T} \frac{p^{2}(t)}{p_{0}^{2}} d t\right]
$$

En la ecuación (3) se puede observar que la energía es integrada en la totalidad del tiempo sin promediación temporal. De este modo, basta que el tiempo de integración sea mayor que la duración del disparo.

\section{PROCEDIMIENTO}

Inicialmente se busca sistematizar el ingreso de datos mediante un código computacional para obtener de manera rápida e intuitiva resultados expresados en tablas y gráficos. En una segunda instancia se realizan mediciones de nivel de exposición sonora en campo libre y se recopila la información de las armas y proyectiles utilizados para luego utilizar como datos de entrada en el modelo computacional. Se comparan los valores globales medidos con los calculados, expresando el resultado como diferencia o error de estimación.

\section{A. Diseño del Código}

Se desarrolla un software de modelado que permita múltiples opciones de ingreso, dependiendo de los datos que posea el usuario acerca del arma y la munición a utilizar. De este modo se puede realizar desde una estimación sencilla con muy poca información de entrada utilizando coeficientes normalizados, hasta un cálculo detallado permitiendo al usuario definir todos la información que posea.

Una vez especificados los valores asociados al propelente se debe indicar el tipo de arma a utilizar, su distribución energética (habitualmente esférica) y el ángulo de incidencia de interés. Independientemente del ángulo indicado para el cálculo de nivel de exposición sonora, el software realiza el cálculo de la energía angular de la fuente para todos los ángulos y así se obtiene un patrón polar de directividad. Es requerido ingresar el tipo de suelo y la distancia a la fuente a la que se encuentra el receptor.

Se computa nivel de exposición sonora tanto global (con ponderación A y Z) como por bandas de tercios de octava desde $12.5 \mathrm{~Hz}$ hasta $10 \mathrm{kHz}$.

\section{B. Mediciones Acústicas}

Se busca medir el nivel de exposición sonora de dos armas de topologías bien diferentes: una pistola calibre 9 $\mathrm{mm}$ y una escopeta calibre $12 / 70$. Ambas son operadas por personal calificado y debidamente acreditado para dicha tarea.

Para realizar la medición se siguen los lineamientos establecidos en la ISO 17201-1 [11]. El sitio de medición es 
de tierra y pasto sin la presencia de objetos verticales en un radio de 30 metros y cuenta con todas las medidas de seguridad necesarias para ejecutar disparos. Se define un punto donde se ubica el disparador y este apunta hacia un único blanco al frente sobre un banco de arena, estableciendo una orientación de tiro determinada. Alrededor de dicho eje, se sitúa una grilla con puntos de medición de manera concéntrica a 21 metros de distancia de la fuente, como se muestra en la Fig. 1. Se asume simetría de la distribución energética respecto de la línea de disparo y se establecen puntos de medición desde $30^{\circ}$ hasta $180^{\circ}$ cada $15^{\circ}$ delante de la línea de fuego y cada $30^{\circ}$ detrás de esta. El punto de medición más cercano a la línea de disparo se ubica a $30^{\circ}$ de esta por cuestiones de seguridad. La posición adoptada por el disparador es de tipo "Weaver".

Se utiliza un medidor de sonido tipo 1 con análisis por bandas de tercios de octava debidamente calibrado y certificado según las normas nacionales de aplicación. El tiempo de integración se configura en Fast y se registran los niveles de exposición sonora. El equipo de medición cuenta con un protector anti-viento y se ubica sobre un trípode a 1.5 metros sobre el nivel del suelo, al igual que el arma al momento de ser disparada. Se verifica que en ninguna posición se supere el nivel de saturación de micrófono del equipo establecido en $137 \mathrm{dBA}$.

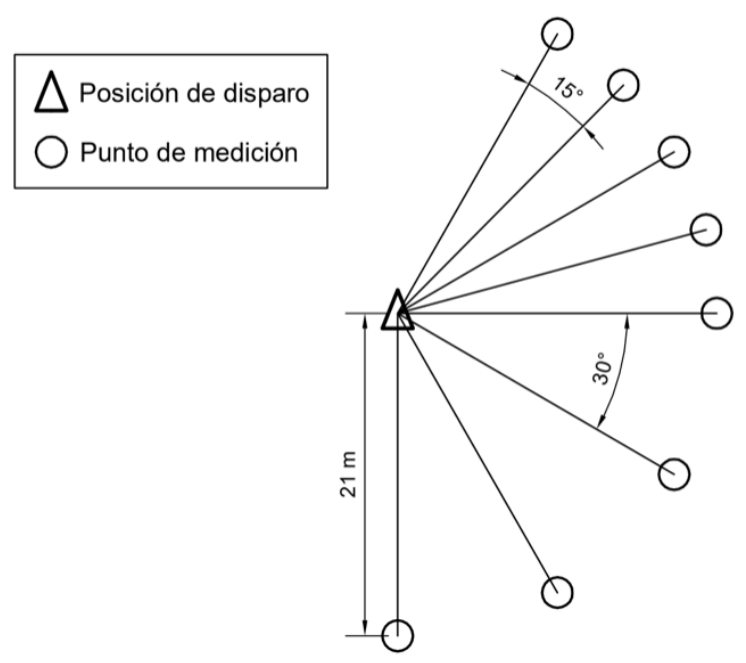

Fig. 1. Vista sperior de la grilla de puntos de medición alrededor de la posición de disparo.

Se realizan 3 repeticiones en cada posición para obtener un valor promedio y el desvío estándar.

\section{RESULTADOS}

A modo de ejemplo, se presenta a continuación el cómputo obtenido para la distribución de la energía angular de la fuente por bandas de tercios de octava en un ángulo determinado, en este caso el ángulo es de $90^{\circ}$, perpendicular a la línea de disparo, para la pistola (Fig. 2) y para la escopeta (Fig. 3). Es posible obtener gráficos similares para distintos ángulos de interés.

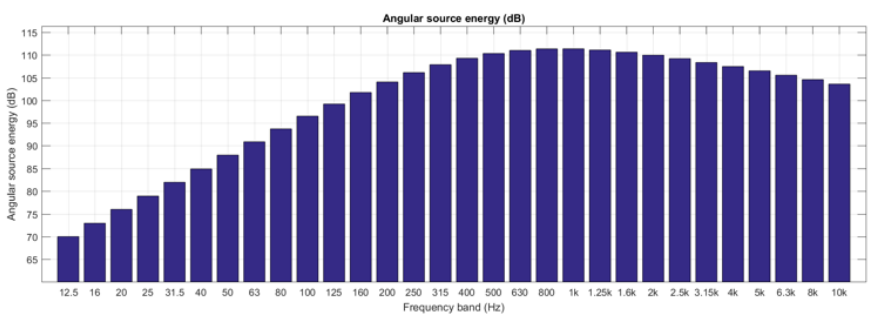

Fig. 2. Estimación de la distribución de la energía angular de la fuente por bandas de tercios de octava para la pistola bajo estudio a $90^{\circ}$.

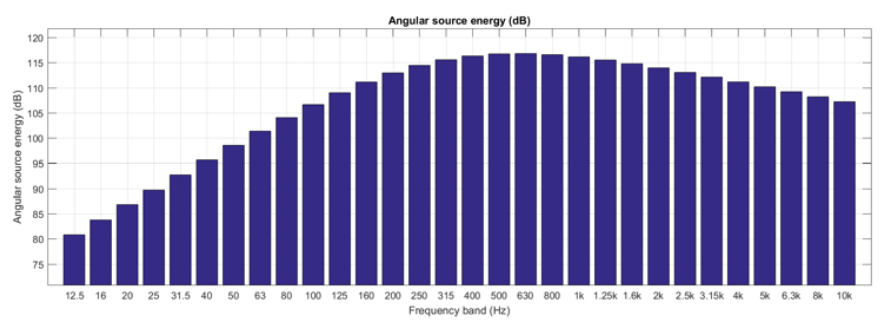

Fig. 3. Estimación de la distribución de la energía angular de la fuente por bandas de tercios de octava para la escopeta bajo estudio a $90^{\circ}$.

A modo de validación del presente código, se comparan los niveles de exposición sonora globales calculados con los medidos en cada uno de los ángulos estudiados para cada configuración arma-munición. En la Tabla I y Fig. 4 se presenta la diferencia de los niveles obtenidos entre el modelo calculado y las mediciones acústicas para la pistola calibre $9 \mathrm{~mm}$. Cabe destacar que cada valor utilizado como "medido" para la comparación es resultado del promedio entre las 3 repeticiones realizadas. Asimismo se muestra el desvío estándar de la media obtenido para cada situación.

TABLA I

DIFERENCIA ENTRE VALORES CALCULADOS Y MEDIDOS PARA LA PISTOLA CALIBRE 9 MM Y DESVÍO ESTÁNDAR DE LA MEDICIÓN.

\begin{tabular}{|c|c|c|}
\hline Ángulo & Diferencia (dB) & Desvío estándar (dB) \\
\hline 30 & -0.9 & 0.31 \\
\hline 45 & -1.1 & 0.42 \\
\hline 60 & -1.5 & 0.06 \\
\hline 75 & -2 & 0.55 \\
\hline 90 & -1.5 & 0.92 \\
\hline 120 & 0.4 & 0.61 \\
\hline 150 & -3.6 & 0.9 \\
\hline 180 & -4.9 & 0.31 \\
\hline
\end{tabular}

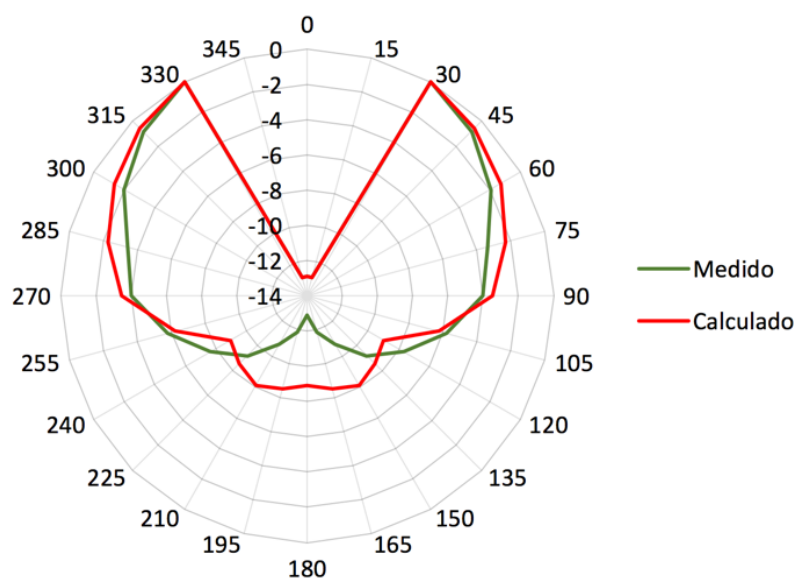

Fig. 4. Patrón polar de directividad de la pistola calibre $9 \mathrm{~mm}$ según el modelo de cálculo y las mediciones acústicas. 
En la Tabla II y Fig. 5 se presenta la diferencia de los niveles obtenidos entre el modelo calculado y las mediciones acústicas para la escopeta calibre 12/70 junto con su desvío asociado.

TABLA II

DIFERENCIA ENTRE VALORES CALCULADOS Y MEDIDOS PARA LA ESCOPETA CALIBRE 12/70 Y DESVÍO ESTÁNDAR DE LA MEDICIÓN.

\begin{tabular}{|c|c|c|}
\hline Ángulo & Diferencia (dB) & Desvío estándar (dB) \\
\hline 30 & 1 & 0.47 \\
\hline 45 & 0.5 & 0.64 \\
\hline 60 & 0.1 & 1.24 \\
\hline 75 & -0.1 & 0.29 \\
\hline 90 & -0.6 & 0.96 \\
\hline 120 & -0.8 & 0.8 \\
\hline 150 & -1.1 & 1.55 \\
\hline 180 & -0.1 & 2.08 \\
\hline
\end{tabular}

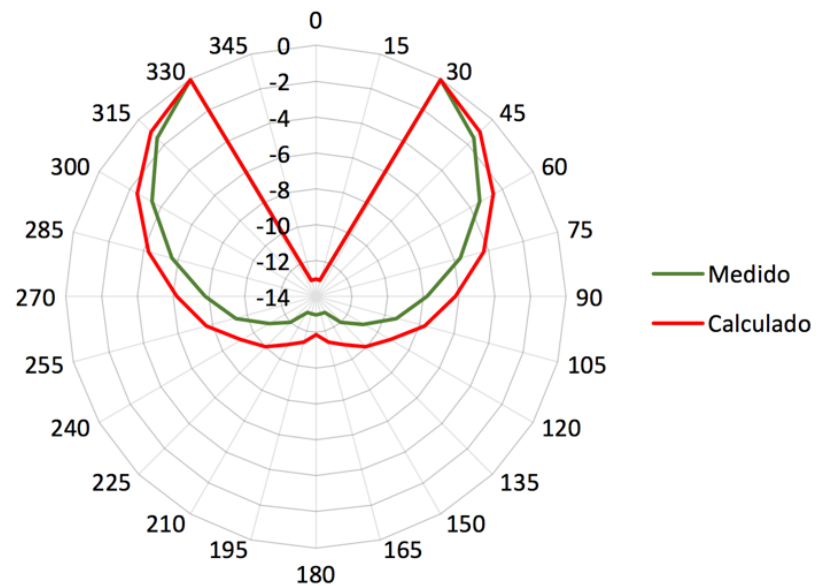

Fig. 5. Patrón polar de directividad de la escopeta calibre 12/70 según el modelo de cálculo y las mediciones acústicas.

\section{CONCLUSIONES}

Respecto de los valores que se calcularon, se logra validar el modelo computacional implementado con un muy buen nivel de estimación (error menor a $1.1 \mathrm{~dB}$ ) para el caso de una escopeta calibre 12/70. Para el caso de la pistola calibre $9 \mathrm{~mm}$ la estimación es buena (error menor a los $2 \mathrm{~dB}$ ) delante de la línea de tiro pero presenta una mayor discrepancia detrás del disparador (error de hasta $4.9 \mathrm{~dB}$ ).

Por otro lado, se puede observar que el patrón direccional de la pistola como resultado de las mediciones se asemeja al patrón obtenido para la escopeta. Esto sugiere la posibilidad de revisar la corrección direccional de la pistola debido al tipo de arma y los coeficientes empíricos involucrados, considerando que el modelo posea una sobreestimación del efecto de difracción debido al disparador. Cabe destacar que el desvío en los puntos de mayor error obtenido $\left(150^{\circ} \mathrm{y}\right.$ $180^{\circ}$ ) es de $0.9 \mathrm{~dB}$ y $0.31 \mathrm{~dB}$ respectivamente, de modo que no se cree que la mencionada diferencia se deba a falencias de la medición.

Por último, se puede afirmar que es posible llevar a cabo una medición acústica de disparos de armas de fuego en Argentina de acuerdo a normativas internacionales $y$ obtener resultados confiables (desvío estándar máximo de $2.08 \mathrm{~dB}$ ) con recursos muy limitados.

\section{AGRADECIMIENTOS}

El presente trabajo ha contado con el apoyo institucional de Gendarmería Nacional Argentina. Se agradece especialmente al Comandante General Orlando Livio Caballero, Comandante Mayor Hugo Ariel Iseas, Escuela de Oficiales General Don Martín Miguel de Güemes, Silvia Cabeza, Mariano Seratín y Agustín Arias.

\section{REFERENCIAS}

[1] M. Brink y J. M. Wunderli, "A field study of the exposureannoyance relationship of military shooting noise,"J. Acoust. Soc. Am., vol. 127, pg. 2301-2311, Abril 2010.

[2] S. Kajander y A. Parri, "Management of the environmental impact of shooting ranges," Best Available Techniques, The Finnish Enviroment 4/2014, Ministerio de Medioambiente, Finlandia.

[3] R. C. Maher y S. R. Shaw, "Directional aspects of forensic gunshot recordings," AES $39^{\text {th }}$ International Conference, Hillerod, Dinamarca, Junio 2010.

[4] R.G. Pregliasco y E. N. Martínez, "Estudio acústico de un homicidio," II Seminario Regional de Física Forense, Bariloche, Argentina, 2001.

[5] T. Markula, "Propagation, measurement and assessment of shooting noise," M. tesis, Universidad Politécnica de Helsinki, Finlandia, 2006

[6] ISO 17201-2:2006, "Acoustics - Noise from shooting ranges - Part 2: Estimation of muzzle blast and projectile sound by calculation," International Organization for Standardization, 2006.

[7] K W. Hirsch, "Estimation of acoustical source strength of muzzle blasts on the basis of launch speed and bullet weight," Internoise 99, Florida, Estados Unidos, Diciembre 1999.

[8] W. Weber, "Das Schallspektrum von Knallfunken und Knallpistolenmiteinem Beitragüber die Anwendungsmöglichkeiten in derelektroakustischen Messtechnik," Akustische Zeitschrift 4, pg. 377-391, 1939.

[9] ISO 17201-3:2010, “Acoustics - Noise from shooting ranges - Part 3: Guidelines for sound propagation calculations," International Organization for Standardization, 2010.

[10] ISO 9613-2:1996, "Acoustics - Attenuation of sound during propagation outdoors - Part 2: General method of calculation," International Organization for Standardization, 1996.

[11] ISO 17201-1:2005, "Acoustics - Noise from shooting ranges - Part 1: Determination of muzzle blast by measurement," International Organization for Standardization, 2005. 